\title{
A Review of Mathematical Knowledge Management ${ }^{\star}$
}

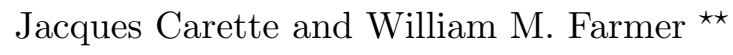 \\ Department of Computing and Software \\ McMaster University \\ Hamilton, Ontario, Canada
}

\begin{abstract}
Mathematical Knowledge Management (MKM), as a field, has seen tremendous growth in the last few years. This period was one where many research threads were started, and the field was defining itself. We believe that we are now in a position to use the MKM body of knowledge as a means to define what MKM is, what it worries about, etc. In this paper, we review the literature of MKM and gather various metadata from these papers. After offering some definitions surrounding MKM, we analyse the metadata we have gathered from these papers, in an effort to cast more light on the field of MKM and its evolution.
\end{abstract}

\section{Introduction}

In 2001 Bruno Buchberger and Olga Caprotti organized the First International Workshop on Mathematical Knowledge Management [6, 7] which was held September 24-26, 2001 at the Research Institute for Symbolic Computation (RISC) in Hagenberg, Austria. The MKM 2001 workshop, attended by 60 or so participants from 10 countries, launched the field of Mathematical Knowledge Management $(\mathrm{MKM})^{1}$ and was the first in a series of international $[6,7,2,1,21,4,20,3]$ and regional [23-26] conferences and workshops on MKM. Since its inception, the MKM community has struggled with questions like "What does it mean to manage mathematical knowledge?", "What should the field of MKM be?", "Should MKM have a wide focus?", if not, "What topics should MKM focus on?", "In what direction is MKM heading?", and "Is MKM making progress?". We agree with those who point out that this field is about "(MK)M" rather than "M(KM)".

\footnotetext{
* This research was supported by NSERC.

$\star \star$ carette, wmfarmer\} @mcmaster.ca.

${ }^{1}$ We will use "MKM" exclusively to mean the field of Mathematical Knowledge Management that started with MKM 2001 and "mathematical knowledge management" to mean the activity of managing mathematical knowledge that started centuries before MKM 2001.
} 
In this paper we seek to answer these and similar questions by reviewing the literature of MKM, particularly the papers presented at the previous seven international MKM conferences (MKM 2001, 2003, 20048). By gathering and analyzing various metadata about the MKM papers of the past we would like to show how where MKM is today, and lay the groundwork for future work that can trace its evolution.

Our aim with this paper is both to survey the current state of MKM and to give future surveyors clear data (and hopefully a clear analysis) of the beginnings of MKM. We also want to offer a tested framework for classifying and analyzing future MKM research.

In the next section, we cover our understanding of MKM, and in section 3 we review the history of "mathematical knowledge management", as a survey of the context in which we understand the field. In section 4, we outline our data gathering and data analysis methodology. In the following section, we lay out the raw results we have obtained, and in section 6 we analyze them. We close with a conclusion.

\section{What is MKM?}

In 2004 in the article [14], we described MKM as follows:

MKM is a new interdisciplinary field of research in the intersection of mathematics, computer science, library science, and scientific publishing. The objective of MKM is to develop new and better ways of managing mathematical knowledge using sophisticated software tools. MKM is expected to serve mathematicians, scientists, and engineers who produce and use mathematical knowledge; educators and students who teach and learn mathematics; publishers who offer mathematical textbooks and disseminate new mathematical results; and librarians and mathematicians who catalog and organize mathematical knowledge.

Although mathematical knowledge possesses several characteristics that sharply distinguish it from other kinds of knowledge, MKM also has a nontrivial intersection with the field of general knowledge management [15].

MKM is indeed a new field of research, but mathematicians have been concerned with managing mathematical knowledge for hundreds, if not thousands, of years. A short history of mathematical knowledge management is given in the next section. However, mathematical knowledge management is now a much greater concern to mathematicians and other mathematics practitioners than it ever was before. There are several reasons for a new heightened interest in managing mathematical knowledge. 
First, since World War II there has been an explosion in the mathematical knowledge produced by mathematicians. The evidence for this statement is abundant. One only has to examine the growth in mathematics articles, reviews, journals, conferences, etc.

Second, there has also been a parallel explosion in the mathematical knowledge produced by scientists and engineers as a by-product of their work. Perhaps the best example of this explosive growth is seen in software development. Computer scientists and software engineers produce millions of software artifacts - requirements specifications, design documents, pieces of computer code - that are essentially mathematical objects. The development and analysis of these artifacts generates an overwhelming amount of highly specific, but still quite valuable, mathematical knowledge.

Third, due to the rise in computer and communication systems, how mathematical knowledge is managed - that is, articulated, organized, disseminated, and accessed - is in the midst of a profound transformation. One example is that a large, and quickly growing, body of mathematical knowledge is now represented either axiomatically by logical theories or algorithmically by symbolic computation programs. Another example is the many new ways that mathematical knowledge is being disseminated, particularly involving the web.

The field of MKM was established to address the large and increasing need for effective mathematical knowledge management. In the eight years since MKM 2001, researchers have approached the task of managing mathematical knowledge from different points of view and have pursued different topics. It is our contention that the collection of these views and topics is a strong indication of what MKM is and where it is heading. Consequently, our review will focus on extracting from the MKM literature the dominant MKM views and topics.

\section{History}

While mathematical knowledge management has been named as a separate endeavor only recently, its history goes back much further at least to Euclid's great and extraordinarily influential Elements.

For the formalist, certainly one important milestone is Frege's Begriffsschrift [16], to whom we owe modern logic. In Hilbert's hands, this became his famous Program, while Russell and Whitehead produced the Principia Mathematica [31], to which we owe type theory. While Gödel's incompleteness theorem [17] certainly put an understandable damper on 
these developments, luckily many nevertheless persevered. Of course, one must mention the Bourbaki project as extolling the virtues of a formal library of mathematics.

But Bourbaki was hardly the first to try to design such a library. Leibnitz, frequently credited as having founded both library science and information theory [10], deserves first-mover credit here. The issues of managing large amounts of information (including substantial parts of mathematics) were already brough to the fore by Denis Diderot's Encyclopédie ou dictionnaire raisonné des sciences, des arts et des métiers [12].

Other aspects of mathematical knowledge management have a similarly extended history. Those interested in mathematical presentation would be well advised to read Cajori's monumental 1929 A History of Mathematical Notations [9]. For the ones more concerned with interactivity, watching Douglas Engelbart's 1968 Mother of All Demos [13] is humbling.

For those most interested in mechanizing mathematics, it is well worth revisiting the early pioneers like Turing and von Neumann (in particular [29] ). Completely indispensable is a thorough reading of the $\mathrm{Au}$ tomath papers $[11,27]$ — some recent MKM work just "rediscovers" some of de Bruijn's early insights. Similarly, the QED Manifesto [5] has helped frame the discussion around formalized mathematics for a very long time (see [32] as an enlightening and readable example).

The more recent history of many parts of MKM have been covered elsewhere (although a unified treatment is still missing), and we will not repeat that here. However, we felt that it was important to remind our readers that mathematical knowledge management actually has a very long history, if one just knows where to look. This history is for us the proper context in which to evaluate the recent work explicitly labeled as Mathematical Knowledge Management.

\section{Methodology}

Before writing this paper, we first agreed on the methodology we should follow. First and foremost, although our results will inevitably be colored by some of our biases, we wanted our results to reflect the field itself. This meant that we have to carefully follow a bottom-up data gathering process where we would systematically review the MKM literature for metadata.

We decided that the refereed proceedings of the previous seven international MKM conferences should be considered the "primary sources". 
The refereeing process serves two purposes: insuring a minimal level of quality as well as asserting that the contributions are "on topic". While there are secondary sources of useful information on MKM, choosing amongst these would have required too much subjective judgment on our part. We will come back to this issue in a later section.

More specifically, this meant that we had to review all 143 papers contained in $[7,2,1,21,4,20,3]$ (which also contain papers for co-located conferences but which are not counted here). A first pass was done to extract the main "topics" which were discussed in every paper. Although at least one of us has looked through every page of every paper (more than once!), we relied heavily on the abstract to extract these "topics". We then formed groups of topics which seemed closely related and came up with labels and descriptions for each of these ${ }^{2}$. At no point did we ever discuss whether any topic was important (or not), interesting (or not), relevant, etc. When abstracting from the specifics to get general topics, the only criterion was: Is "mathematical knowledge" a crucial aspect? In some cases, for example issues relating to distributed systems, we decided that the topic (as it appeared in the papers under review) was core computer science rather than containing specific MKM issues.

As we still ended up with a rather long list of topics, it was natural to try to organize the list somehow. At first, we naïvely attempted to create a hierarchy ${ }^{3}$ out of these topics - and failed miserably. This is when we realized that we were oversimplifying the problem and, firmly inspired by the field of software architecture, we saw that these papers differed not only in their topics, but also in their points of view. The next section explains this in more detail. We then had to re-review each paper to extract the author's point-of-view, as this information could not be obtained from the list of topics.

\section{Results}

This section presents the results of our investigation of the MKM literature. More specifically, we present the points of view, topics and quantitative data relating to these.

\footnotetext{
${ }^{2}$ Although we believe the extraction of important topics was objective, the grouping and labeling is inevitably somewhat more subjective.

${ }^{3}$ Especially naïve as both of us had read [28].
} 


\begin{tabular}{|c|c|c|c|c|}
\hline Document & Library & Formal & Digital & Interactive Process \\
\hline
\end{tabular}

\section{$5.1 \quad$ Views}

Fig. 1: The 6 views of MKM

In our investigation of the MKM literature we identified six major lenses through which researchers view MKM. These views are not incompatible; more than one view is often exhibited in the same research paper.

1. Document. Mathematical knowledge is traditionally communicated via mathematical documents. The document view of MKM sees the management of mathematical knowledge as largely happening inside documents, and managing these documents is a central concern. The documents, however, can have several forms. Some examples are articles in journals, hypertext documents on the web, and theory files produced using theorem provers. An example of a recent MKM 2008 paper written from the document view is "On Correctness of Mathematical Texts from a Logical and Practical Point of View" by K. Verchinine et al. [30]. It is concerned with formalized mathematical documents.

2. Library. One major view of mathematics is that it is a huge body of mathematical facts. According to the library view of MKM, the main objective of MKM is to design and implement libraries, repositories, and archives in which a part of the body of mathematical facts is assembled, organized, and made accessible in various ways. How a mathematical library works is the primary concern; what is held in a library and how it is represented are secondary concerns. The MKM 2008 paper "Cross-Curriculum Search for Intergeo" by P. Libbrecht [22] takes a library view of MKM. It describes how a library of interactive geometry resources is organized so that it facilitates search.

3. Formal. Mathematical knowledge is highly structured and interrelated. In the formal view of MKM, mathematical knowledge is managed according to how it is structured and interrelated. Deduction and computation are a very important part of this view since they are the principal means by which the structure of mathematical knowledge is created, discovered, and communicated. A formal view is taken in the MKM 2007 paper "Formal Representation of Mathematics in a Dependently Typed Set Theory" by F. F. Horozal and C. E. Brown [19]. It studies the relationship between an informal presentation of introductory real analysis and a formal presentation of it in the Scunak type theory. 
4. Digital. Like almost all other kinds of knowledge, there is a strong impetus to digitize mathematical knowledge so that it can be handled by computer and communication systems. The digital view of MKM considers the essence of managing mathematical knowledge to be managing digital objects that encode mathematical knowledge. The digital view, in particular, is concerned with how mathematical knowledge can be put on and accessed via the web. A. S. Youssef's MKM 2007 paper "Methods of Relevance Ranking and Hit-Content Generation in Math Search" [33] takes a digital view. It proposes techniques for searching digital mathematics libraries.

5. Interactive. Mathematical knowledge is created, discovered, and communicated by human-to-human and human-to-tool interaction. The basis of the interactive view of MKM is that mathematical knowledge can only be properly managed within the context of this interaction. This view emphasizes the central role of mathematical knowledge in how mathematics is learned, produced, and applied. The MKM 2008 paper "Specifying Strategies for Exercises" by B. Heeren et al. [18] exhibits an interactive view. It investigates the specification of strategies for use in exercise-solving systems.

6. Process. Another major view of mathematics is that it is a process in which mathematical models are created, explored, and interconnected. The process view of MKM focuses on how mathematical knowledge is produced. Managing mathematical knowledge is thus seen as managing the process that produces mathematical knowledge. This view includes a concern for the community of mathematicians, scientists, and engineers who produce mathematical knowledge. Process is the dominant view taken in A. Bundy's MKM 2008 paper " Automated Signature Evolution in Logical Theories" [8]. It argues that logical theories evolve over time and, as a consequence, their signatures need to be managed.

\section{$5.2 \quad$ Topics}

A great many topics have been addressed in the MKM literature. From the topics our investigation has found, we have consolidated a list of 25 topics which the MKM community, through MKM literature, has concerned itself with. It is important to remember that these topics were chosen because some papers made the point that these topics were of special concern for "mathematical knowledge management". 


\begin{tabular}{|lllll||}
\hline Representation & Case-study & Extraction & Markup & Presentation \\
Mechanized & Interactivity & Search & Practice & Web \\
Translation & Organization & Library & Usability & Document \\
Education & Environment & Integrity & Process & Framework \\
Communication Maintenance & Philosophy & Natural- & Publishing \\
& & & language & \\
\hline
\end{tabular}

Fig. 2: The 25 topics of MKM

1. Representation. Techniques and devices for representing mathematical knowledge including data structures, logics, formal theories, normalization, diagrams, etc.

2. Case-study. Work that focuses on a particular example of mathematical knowledge, most often as a requirements gathering and analysis exercise.

3. Mechanized. Systems, such as theorem provers and computer algebra systems, that provide mathematical services that mechanize certain aspects of the mathematics process.

4. Markup. Markup languages for expressing mathematics such as XML, MathML, OpenMath, and OMDoc.

5. Presentation. Techniques and devices for presenting mathematical knowledge (like notation and diagrams).

6. Extraction. Techniques for extracting or inferring mathematical knowledge from mathematical documents and other sources.

7. Search. Searching and querying collections of mathematical knowledge as well as mathematical services.

8. Practice. Today's practice of mathematics by mathematicians, scientists, and engineers including issues like the mathematical vernacular, mathematics communities, and the role of context and convention.

9. Process. The process of creating, discovering, exploring, and applying mathematical knowledge.

10. Translation. The translation of mathematical knowledge from one representation to another, including parsing techniques.

11. Usability. Techniques for making mathematical knowledge more usable.

12. Web. The fundamental use of the web to communicate mathematical knowledge and to support mathematics practice.

13. Organization. The organization of mathematical knowledge, including the use of ontologies and metadata.

14. Natural-language. Mathematical knowledge expressed via natural languages.

15. Library. Libraries, repositories, and archives of mathematical knowledge. 
16. Document. Mathematical documents of all forms.

17. Education. MKM in, and for, mathematical education.

18. Integrity. The consistency, correctness, and certification of mathematical knowledge.

19. Environment. The development and use of software environments for managing mathematical knowledge.

20. Maintenance. The maintenance and version control of collections of mathematical knowledge.

21. Philosophy. The impact of the philosophy of mathematics on MKM.

22. Communication. The communication of mathematical knowledge between systems, particularly heterogeneous systems.

23. Framework. Frameworks for managing mathematical knowledge.

24. Publishing. Issues concerning the publication of mathematical knowledge.

25. Interactivity. Human-to-human and human-to-tool interaction involving mathematical knowledge.

\subsection{Statistics}

In Figure 3, we see the sorted distribution of weighted views for all papers. Each paper is assigned a total weight of 1 , and this weight is divided evenly amongst all points of view espoused by the paper. Figure 4 is the similar histogram for topics. We also looked at the unweighted data, and for both views and topics, the ordering was essentially the same, i.e. the only

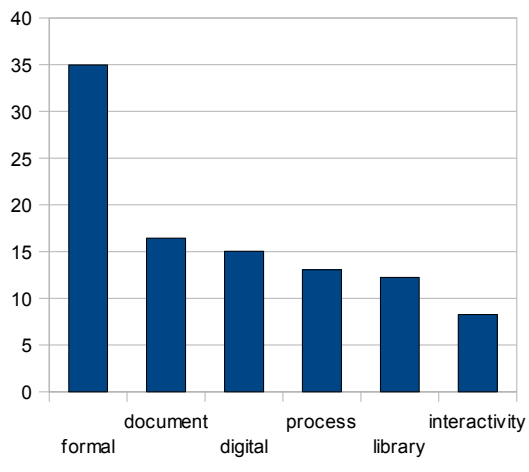

Fig. 3: Views changes were when views/topics already had statistically indistiguishable counts.

We have also broken down the data in these two figures by year, rescaling the results as percentages per year. We can extract some information from the view-per-year data (see Appendix A), but there is not enough data (143 papers in $25 \times 7=175$ bins) to extract meaningful results from a similar breakdown of the topics data. We were unable to find a meaningful clustering of the topics that might allow trends (if any) to become visible. 


\section{Analysis}

What can we extract from this data? It is very clear that the community tends to favor a formal view of mathematics. While that is not totally unexpected, looking that the PROBLEM of MKM, it would probably be healthier if the points of view were more uniformly distributed. Statistically speaking, the document and digital views are tied for second, and process and library third, with interactivity getting the least attention. We believe that the large ratio $(4: 1)$ between formal and interactivity is mainly due to the current makeup of the community (many coming from formal backgrounds and otherwise working on highly mathematical problems) and the current state of the field (it is difficult to build a novel interactive system atop quicksand and convince formalists of its worth). In between, considering the amount of time and energy it takes to build a reasonable library of mathematics, it is probably unsurprising that this viewpoint has not received equal attention, especially since MKM has not attracted many system builders.

The distribution of topics clearly indicates that representation issues get the highest share of the community's attention (with the related issues surrounding markup joining in at number 4). More interesting is the secondplace showing of case-study: we take this as a sign of a burgeoning field which takes the scientific method seriously and is doing some amount of requirements analysis before diving in with solutions ${ }^{4}$.

We can also analyze the correlations between views (seen as depending on the topics) and viceversa (raw data is shown in Appendix B). For the views, the most significant correlation (0.7) is between the library and digital

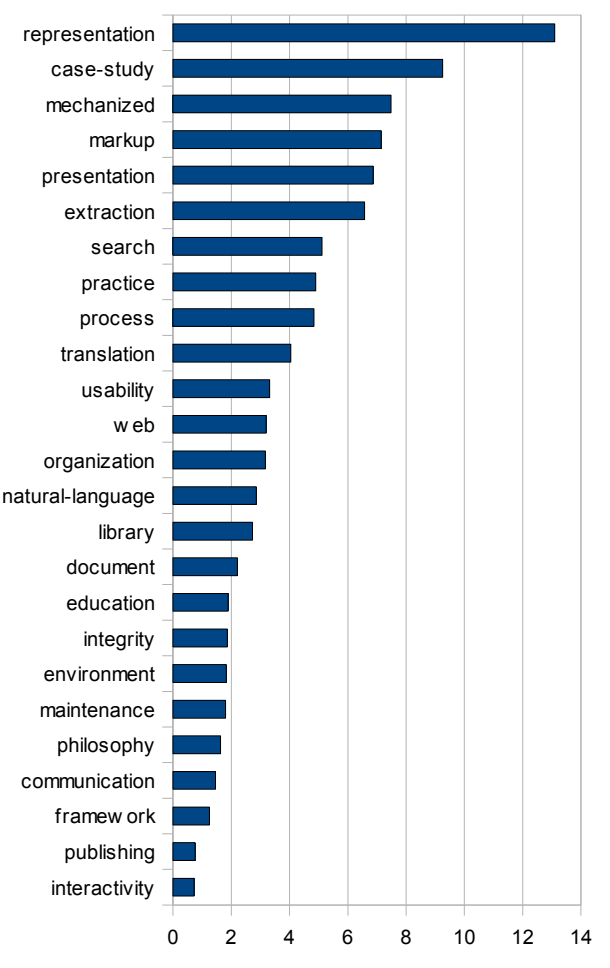

Fig. 4: Topics

\footnotetext{
${ }^{4}$ A lack of requirements analysis very often leads to interesting solutions to problems which did not need solving.
} 
views, which basically says that no one today is looking at large repositories of mathematics outside the digital domain. There is no correlation (0.0) between digital and interactive; this is potentially an artifact of how we chose to assign views, but not clearly so: the emphasis in the digital view is on mathematical knowledge being digital, while the interactive view emphasizes human interaction (most often on computers). It is reassuring that there are no negative correlations, which would have indicated a real flaw in our choices!

Analyzing the correlations between topics, there is a very strong pairwise correlation $(>0.87)$ between the 4 topics representation, casestudy, mechanized and usability. In other words, regardless of point of view, these topics tend to appear together. This can also be interpreted to indicate that MKM has a strong affinity for the topics covered by the Calculemus conference, and would further justify the co-location of these conferences for 2007,2008 and 2009. At the other extreme, the pairs (markup, education), (extraction, education), and (process, web) are strongly negatively correlated $(-0.9,-0.95$ and -0.89 respectively). This also makes sense as, no matter how one looks at MKM topics, neither markup nor techniques for information extraction are (currently) relevant to MKM issues in education, nor is the advances in web technology as discussed in MKM papers (currently) relevant to the process of creating mathematics ${ }^{5}$.

Looking at the per-year data, only a couple of trends appear to be statistically significant: the process view is gaining some traction, while the formal view appears to be slowly losing ground.

\subsection{Secondary sources}

Did we miss something important by ignoring some secondary sources? If we look at the topics and views covered in the different regional workshops and less formal conference proceedings $[6,23-26]$, we see ${ }^{6}$ that this is not the case. In fact, the topics and views of the talks at these other meetings seem to fall even more neatly into our categories than many papers in the MKM proceedings! What we do notice is a different emphasis, with the formal view being less prominent, but otherwise all views and essentially all topics are represented.

\footnotetext{
${ }^{5}$ even though mathematicians routinely use web 1.0 mechanisms as part of the social fabric of creating mathematics

${ }^{6}$ a similar data-gathering effort was done on these sources, but that data was not included in our results
} 


\section{Conclusion}

Our review of the MKM literature has produced a two-dimensional framework based on views and topics for classifying and analyzing MKM research. Although some bias on our part has certainly crept into our analysis, we have made a concerted effort to let the literature speak for itself. Our results show that the MKM community is pursuing a wide range of topics from a reasonably balanced set of view points. Our analysis shows that some trends and correlations are clearly evident such as the persistent interest in the formal view and the strong correlation between the formal view and the representation topic.

What stands out most in this work are the views. MKM researchers take different points of view when they do their research and write their results. The six views we have identified appear to cover, either individually or in combination, the views exhibited in the MKM literature. The views embody the different ways people see mathematical knowledge as well as the different ways people see mathematics itself. Like Parnas [28], we refuse to oversimplify MKM and shoehorn it into a hierarchy. More productive is to frankly embrace its complexity, and try to tame it with

tools appropriate for a complex field rather than to do forensics on a carcass.

\section{References}

1. Andrea Asperti, Grzegorz Bancerek, and Andrzej Trybulec, editors. Mathematical Knowledge Management, Third International Conference, MKM 2004, Bialowieza, Poland, September 19-21, 2004, Proceedings, volume 3119 of Lecture Notes in Computer Science. Springer, 2004.

2. Andrea Asperti, Bruno Buchberger, and James H. Davenport, editors. Mathematical Knowledge Management, Second International Conference, MKM 2003, Bertinoro, Italy, February 16-18, 2003, Proceedings, volume 2594 of Lecture Notes in Computer Science. Springer, 2003.

3. Serge Autexier, John Campbell, Julio Rubio, Volker Sorge, Masakazu Suzuki, and Freek Wiedijk, editors. Intelligent Computer Mathematics, 9th International Conference, AISC 2008, 15th Symposium, Calculemus 2008, 7th International Conference, MKM 2008, Birmingham, UK, July 28 - August 1, 2008. Proceedings, volume 5144 of Lecture Notes in Computer Science. Springer, 2008.

4. Jonathan M. Borwein and William M. Farmer, editors. Mathematical Knowledge Management, 5th International Conference, MKM 2006, Wokingham, UK, August 11-12, 2006, Proceedings, volume 4108 of Lecture Notes in Computer Science. Springer, 2006.

5. R. Boyer. The QED Manifesto. In A. Bundy, editor, Proceedings CADE 12, pages 238-251, 1994. 
6. Bruno Buchberger and Olga Caprotti, editors. Electronic Proceedings of the First International Workshop on Mathematical Knowledge Management: MKM 2001. RISC-Linz, 2001. Available at http://www.risc.uni-linz.ac.at/about/ conferences/MKM2001/Proceedings.

7. Bruno Buchberger, Gaston H. Gonnet, and Michiel Hazewinkel, editors. Mathematical Knowledge Management, volume 38 of Ann. Math. Artif. Intell. Kluwer, 2003.

8. Alan Bundy. Automating signature evolution in logical theories. In Autexier et al. [3], pages 333-338.

9. Florian Cajori. A History of Mathematical Notations. Dover (reprint), 1929.

10. Martin Davis. The Universal Computer: The Road from Leibniz to Turing. W. W. Norton \& Co., Inc., New York, NY, USA, 2000.

11. N.G. de Bruijn. A Survey of the Project Automath. To H.B. Curry: Essays on Combinatory Logic, pages 579-606, 1980.

12. Encyclopédie ou dictionnaire raisonné des sciences, des arts et des métiers, 17511772 .

13. Douglas Engelbart. A research center for augmenting human intellect (demo). http://en.wikipedia.org/wiki/The_Mother_of_All_Demos, December 1968.

14. W. M. Farmer. MKM: A new interdisciplinary field of research. ACM SIGSAM Bulletin, 38:47-52, 2004.

15. W. M. Farmer. Mathematical knowledge management. In D. G. Schwartz, editor, Encyclopedia of Knowledge Management, pages 599-604. Information Science Reference, 2005.

16. Gottlob Frege. Begriffsschrift: eine der arithmetische nachgebildete Formelsprache des reinen Denkens. L. Nebert, Halle a/S, 1879/1997.

17. Kurt Gödel. Über formal unentscheidbare sätze der principia mathematica und verwandter systeme. Monatshefte für Mathematik und Physik, 38(1):173-198, 1931.

18. Bastiaan Heeren, Johan Jeuring, Arthur van Leeuwen, and Alex Gerdes. Specifying strategies for exercises. In Autexier et al. [3], pages 430-445.

19. Feryal Fulya Horozal and Chad E. Brown. Formal representation of mathematics in a dependently typed set theory. In Kauers et al. [20], pages 265-279.

20. Manuel Kauers, Manfred Kerber, Robert Miner, and Wolfgang Windsteiger, editors. Towards Mechanized Mathematical Assistants, 14th Symposium, Calculemus 2007, 6th International Conference, MKM 2007, Hagenberg, Austria, June 27-30, 200\%, Proceedings, volume 4573 of Lecture Notes in Computer Science. Springer, 2007.

21. Michael Kohlhase, editor. Mathematical Knowledge Management, 4th International Conference, MKM 2005, Bremen, Germany, July 15-17, 2005, Revised Selected Papers, volume 3863 of Lecture Notes in Computer Science. Springer, 2006.

22. Paul Libbrecht, Cyrille Desmoulins, Christian Mercat, Colette Laborde, Michael Dietrich, and Maxim Hendriks. Cross-curriculum search for intergeo. In Autexier et al. [3], pages 520-535.

23. Mathematical Knowledge Management Symposium. Web site at http://www. macs.hw.ac.uk/ fairouz/mkm-symposium03/.

24. A North American Workshop on Mathematical Knowledge Management (NAMKM 2002). Web site at http://imps.mcmaster.ca/na-mkm-2002/.

25. Second North American Workshop on Mathematical Knowledge Management (NAMKM 2004). Web site at http://imps.mcmaster.ca/na-mkm-2004/.

26. Workshop on Mathematical Knowledge Management: Sustainability, Scalability and Interoperability. Web site at http://projects.cs.dal.ca/ddrive/seminars/ $\mathrm{mkm} \cdot \operatorname{shtml}$. 
27. R. Nederpelt, J. Geuvers, R. de Vrijer, and eds. Selected Papers on Automath. Studies in Logic and the Foundations of Mathematics 133, 1994.

28. David L. Parnas. On a "buzzword": hierarchical structure, pages 429-440. SpringerVerlag New York, Inc., New York, NY, USA, 2002.

29. Alan M. Turing. Practical forms of type theory. Journal of Symbolic Logic, 13:8094, 1948 .

30. Konstantin Verchinine, Alexander V. Lyaletski, Andrey Paskevich, and Anatoly Anisimov. On correctness of mathematical texts from a logical and practical point of view. In Autexier et al. [3], pages 583-598.

31. A.N. Whitehead and B. Russell. Principia Mathematica. Cambridge University Press, 1910.

32. Freek Wiedijk. The qed manifesto revisited. Studies in Logic, Grammar and Rhetoric, 10(23):121-133, 2007.

33. Abdou Youssef. Methods of relevance ranking and hit-content generation in math search. In Kauers et al. [20], pages 393-406.

\section{A View by Year data}

Percentage of weighted papers for each view, per year.

\begin{tabular}{|l|l|l|l|l|l|l|l|}
\hline & 2001 & 2003 & 2004 & 2005 & 2006 & 2007 & 2008 \\
\hline formal & 39.4 & 35.3 & 37.8 & 28.2 & 54.5 & 19 & 32.5 \\
\hline document & 12.1 & 16.7 & 23.1 & 26.3 & 2.27 & 9.52 & 20 \\
\hline digital & 19.7 & 31.4 & 11.5 & 11.5 & 9.09 & 9.52 & 20 \\
\hline process & 4.55 & 5.88 & 10.9 & 12.8 & 13.6 & 23.8 & 15 \\
\hline library & 15.2 & 8.82 & 8.97 & 13.5 & 11.4 & 23.8 & 5 \\
\hline interactivity & 9.09 & 1.96 & 7.69 & 7.69 & 9.09 & 14.3 & 7.5 \\
\hline
\end{tabular}

\section{B View-Topic data}

Total number of weighted papers for each combination of view and topic. 


\begin{tabular}{|l|l|l|l|l|l|l|}
\hline & formal & document & digital & process & library & interactive \\
\hline representation & 22 & 7.3 & 3.8 & 6.5 & 4 & 3.8 \\
\hline case-study & 16 & 2 & 4.5 & 5.3 & 6.3 & 3.5 \\
\hline mechanized & 18 & 1.5 & 0 & 2.8 & 1.3 & 6 \\
\hline markup & 6.2 & 6.2 & 7.7 & 1.8 & 3.3 & 2.8 \\
\hline presentation & 6.3 & 7.3 & 3.5 & 0.83 & 3.5 & 4.5 \\
\hline extraction & 7.5 & 3 & 6.5 & 1 & 3.5 & 0.5 \\
\hline search & 2 & 0.5 & 7.5 & 2.5 & 6 & 0.5 \\
\hline practice & 4 & 4.5 & 2.5 & 5.5 & 0.5 & 1 \\
\hline process & 2.5 & 0.5 & 0.5 & 0 & 1 & 2.5 \\
\hline translation & 0.5 & 1.5 & 0.5 & 0 & 0 & 1.5 \\
\hline usability & 4.8 & 1 & 3 & 3.3 & 1.8 & 0 \\
\hline web & 5.5 & 5 & 0.5 & 5 & 1.5 & 2.5 \\
\hline organization & 2 & 2 & 3 & 1 & 0 & 0 \\
\hline natural-language & 0.83 & 6.2 & 0.83 & 0.33 & 0.83 & 0 \\
\hline library & 7 & 4.5 & 3 & 0 & 0.5 & 0 \\
\hline document & 1.3 & 0 & 0.33 & 1 & 1.3 & 3 \\
\hline education & 3.5 & 3.5 & 0.5 & 0 & 0.5 & 0 \\
\hline integrity & 1 & 1.5 & 0.5 & 0.5 & 1.5 & 0 \\
\hline environment & 5.5 & 1 & 1 & 2 & 0 & 1.5 \\
\hline maintenance & 1 & 0 & 0 & 3 & 0 & 0 \\
\hline philosophy & 1.3 & 1.2 & 6 & 1.5 & 2.7 & 3.3 \\
\hline communication & 4.2 & 0.33 & 3.2 & 0.33 & 4 & 0 \\
\hline framework & 1 & 2 & 0 & 1 & 2 & 3 \\
\hline publishing & 0 & 1.3 & 0.33 & 0 & 1.3 & 0 \\
\hline interactivity & 0 & 1 & 0 & 3 & 1.5 & 3.5 \\
\hline
\end{tabular}

